\title{
Aislamiento e identificación de Vibrio parahaemolyticus O3:K6 en pescados y moluscos bivalvos procedentes de un mercado pesquero de Lima, Perú.
}

\author{
Isolation and identification of Vibrio parahaemolyticus O3:K6 in fish and bivalve mollusks of a fish market \\ from Lima, Perú.
}

Rocío Aliaga $^{1}$, Jacqueline Miranda ${ }^{2}$, Jesús Zevallos ${ }^{3}$

\begin{abstract}
RESUMEN
Objetivo: Determinar la presencia de Vibrio parahaemolyticus serotipo O3:K6 en pescados y moluscos bivalvos procedentes de un mercado pesquero de Lima. Material y Métodos: Se analizaron 254 muestras entre pescados y moluscos bivalvos siguiendo la técnica descrita por Yukiko Harakudo et al, que consta de un paso de enriquecimiento, siembra en medio cromogénico, evaluación de fermentación de glucosa y la prueba de tolerancia a sales. Las cepas identificadas como Vibrio parahaemolyticus fueron serotipificadas usando anticuerpos monoclonales y evaluadas en su potencial patogénico en medio de Wagatsuma. Resultados: Se aislaron 15 cepas de V. parahaemolyticus, $9(7,5 \%)$ en pescados y $6(4,5 \%)$ en moluscos bivalvos. Tres $(20 \%)$ presentaron fenómeno de Kanagawa positivo indicando la producción de la hemolisina termoestable directa (TDH). Una cepa TDH-positiva aislada en una muestra pescado fue serotipo O3:K6. Conclusiones: Vibrio parahaemolyticus se encuentra distribuido en los recursos hidrobiológicos estudiados con mayor frecuencia en pescados, a partir de los cuales se encontró el serovar O3:K6. Este es el primer reporte de la cepa pandémica O3:K6 de Vibrio parahaemolyticus aislada de fuente no humana en el Perú. (Rev Med Hered 2010;21:139-145).
\end{abstract}

PALABRAS CLAVE:Vibrio parahaemolyticus, pescados, moluscos bivalvos, gastroenteritis, fenómeno de Kanagawa.

\section{SUMMARY}

Objective: To determine the presence of Vibrio parahaemolyticus serotype O3:K6 in fish and bivalves mollusks of a fish market from Lima. Material and methods: We analyzed 254 samples of fish and bivalve mollusks using the technique described by Yukiko Harakudo et al, consisting of an enrichment step, chromogenic plating, evaluation of glucose fermentation and salt tolerance test. The strains identified as Vibrio

Bióloga, Docente de Tecnología Médica, Universidad Peruana Cayetano Heredia. Lima, Perú.

Tecnóloga Médica, Docente de Tecnología Médica, Universidad Peruana Cayetano Heredia. Lima, Perú.

Egresado de la Escuela de Tecnologia Médica, Universidad Peruana Cayetano Heredia. Lima, Perú. 
parahaemolyticus were serotyped using monoclonal antibodies and evaluated their pathogenic potential on Wagatsuma agar. Results: 15 strains of V. parahaemolyticus, $9(7,5 \%)$ in fish and $6(4,5 \%)$ in bivalve mollusks were isolated. $3(20 \%)$ were Kanagawa phenomenon positive indicating the production of thermostable direct hemolysin (TDH). A TDH-positive strain isolated from a fish belonged to the serotype O3: K6. Conclusions: Vibrio parahaemolyticus is distributed in analyzed aquatic resources; most frequently in fish from which was found serovar O3:K6. This is the first report of pandemic O3:K6 strain of Vibrio parahaemolyticus isolated from non-human source in Perú. (Rev Med Hered 2010;21:139-145).

KEYWORDS: Vibrio parahaemolyticus, fish, bivalves mollusks, gastroenteritis, Kanagawa phenomenon.

\section{INTRODUCCIÓN}

El género Vibrio comprende 75 especies, de ellas 12 son consideradas patógenas para el ser humano (1); siendo el Vibrio cholerae el que mayores estragos ha generado a lo largo de las 8 pandemias producidas desde inicios del siglo XIX. El Perú fue azotado por 3 de éstas, la última ocurrida en $1991(2,3)$.

Luego de controlada la epidemia los casos de gastroenteritis por $V$. cholerae se han presentado de forma esporádica; sin embargo, como consecuencia de los cambios climáticos generados por el calentamiento global, hay evidencia del resurgimiento del género Vibrio, presentándose casos de gastroenteritis reemergentes en diferentes partes del mundo, producidos por otra especie del género Vibrio, el V. parahaemolyticus $(4,5)$.

Vibrio parahaemolyticus es una bacteria halofílica, autóctona del ambiente marino, considerada como la principal causa de gastroenteritis bacteriana asociada al consumo de alimentos marinos crudos (6). Pueden causar cuadros de diarrea aguda eventualmente de tipo disentérico, en ocasiones bastante severos y con desenlaces fatales en especial en ciertos grupos poblacionales susceptibles: niños, gestantes, ancianos, inmunocomprometidos, personas con enfermedades hepáticas concomitantes o infección por Helicobacter pylori, entre otras (4).

De un potencial de 71 combinaciones de serotipos $\mathrm{O}: \mathrm{K}$, Vibrio parahaemolyticus O3:K6 es considerado como la cepa pandémica $(5,7,8,9)$ poseedora del gen $t d h$ codificante de la hemolisina termoestable directa (TDH), principal factor de virulencia en esta especie (3), este serotipo a diferencia de los otros es capaz de incrementar rápidamente no sólo la incidencia sino la gravedad de los casos de diarrea aguda en zonas donde se presenten condiciones favorables para su desarrollo.

El fenómeno de "El Niño"; aunado a los cambios climáticos, son condiciones que alteran los parámetros hidrobiológicos normales del ecosistema marino produciendo la multiplicación excesiva de fitoplancton y zooplancton, cuya importancia en la diseminación y persistencia de $V$. cholerae y $V$. parahaemolyticus ya ha sido demostrada $(10,11)$.

La presencia de Vibrio parahaemolyticus (cepas virulentas y avirulentas) total; ha sido usado como indicador en el control de contaminación de alimentos para la prevención de infecciones; sin embargo, la efectividad de esta acción es incierta por la falta de información de la frecuencia de las cepas virulentas en fuentes no humanas en muchos países, así como en el nuestro (12).

Teniendo en cuenta estas consideraciones se realizó el estudio con el objetivo de determinar la presencia de Vibrio parahaemolyticus O3:K6; cepa virulenta, en pescados y moluscos bivalvos de mayor demanda en un mercado pesquero de Lima Metropolitana.

\section{MATERIAL Y MÉTODOS}

La recolección de muestras se llevó a cabo en los meses estivales de enero-febrero del 2009 en los que la temperatura promedio en el litoral peruano fue de $19,85^{\circ} \mathrm{C}$ (13). Se evaluaron 254 muestras ( 120 pescados y 134 muestras de moluscos bivalvos) calculados por la fórmula de prevalencia límite cuya proporción estimada fue obtenida de revisiones bibliográficas $(14,15,16)$.

Los recursos hidrobiológicos para el estudio no presentaban signos de descomposición y se obtuvieron en puestos seleccionados al azar, registrados en el 
mercado pesquero de Ventanilla, Callao, Perú, los que provenían de distintos puertos del litoral. Las especies estudiadas fueron: Trachurus picturatus murphyi (Jurel), Mugil cephalus (Lisa), Odentesthes regia regia (Pejerrey), Aulocomya ater (Choro), Argopecten purpuratus (Concha de abanico) y Gari salida (Almeja) (Tabla 1). Las especies marinas fueron seleccionadas por ser las de mayor consumo por nuestra población, según la Oficina de General de Tecnología de la Información y Estadística del Ministerio de la Producción (17).

Los moluscos tenían las valvas cerradas para asegurar que el aislamiento de Vibrio parahaemolyticus no sea producto de contaminación cruzada. Como medida adicional para evitar la contaminación cruzada entre las especies, las muestras se obtuvieron en bolsas de polietileno, las que fueron trasladadas hasta el laboratorio dentro de contenedores de frío de 4 a $8^{\circ} \mathrm{C}$.

\section{Muestra para el análisis microbiológico}

La muestra de pescado estaba conformada por $25 \mathrm{~g}$ de un homogenizado de la piel y agallas de un espécimen. En el caso de los moluscos bivalvos; la muestra fue constituida por el homogenizado del contenido de 12 moluscos, tomando $25 \mathrm{~g}$, para el análisis de acuerdo a lo descrito en el Manual de Análisis Microbiológico de Administración de Drogas y Alimentos de los Estados Unidos (FDA) (18).

\section{Aislamiento de Vibrio parahaemolyticus}

Se realizó siguiendo el protocolo establecido por Yukiko Hara-Kudo et al (19). Para la serotipificación se utilizaron antisueros compuestos de anticuerpos monoclonales contra los antígenos somático $\mathrm{O} 3$ y capsular K6, y la presencia de la hemolisina termoestable directa (TDH), un factor importante de patogenicidad (20), se realizó mediante la siembra en agar de Wagatsuma para observar el fenómeno de Kanagawa (Gráfico 1).

\section{Análisis estadístico}

Los datos fueron almacenados en una hoja de cálculo del Programa Microsoft EXCEL 2003 y analizados con el paquete estadístico Epi Info (versión 6,04 b). También se calculó la capacidad de detección de $V$. parahaemolyticus serotipo O3:K6 en la población de Odentesthes regia regia (Pejerrey) usando la fórmula de prevalencia límite.

\section{RESULTADOS}

De 254 muestras estudiadas se aislaron 15 cepas de Vibrio parahaemolyticus (5,9\%), de las cuales 9 fueron obtenidas de pescado y 6 a partir de moluscos bivalvos.

Se aisló Vibrio parahaemolyticus en todas las especies de pescado estudiadas, con mayor frecuencia en Odentesthes regia regia (Pejerrey), Aulocomya ater (Choro), en Argopecten purpuratus (Concha de abanico), pero no en almejas (Tabla 2).

En 3/15, ocurrió beta hemólisis (fenómeno de Kanagawa positivo). Solo un aislamiento presentó aglutinación con ambos antisueros monoclonales $\mathrm{O} 3$ y K6, la misma cepa también presentó fenómeno de Kanagawa positivo (cepa pandémica de Vibrio parahaemolyticus O3:K6) (Tabla 2); de esta manera se

Tabla 1. Distribución de muestras según especie y zona de pesca.

\begin{tabular}{clcc}
\hline Grupo biológico* & \multicolumn{1}{c}{ Especie } & Zona de pesca & n \\
\hline \multirow{3}{*}{ Pescados } & Trachurus picturatus murphyi (Jurel) & Tacna & 40 \\
& Mugil cephalus (Lisa) & Bayovar, Paita & 40 \\
& Odontesthes regia regia (Pejerrey) & Pisco, Lomas & 40 \\
Moluscos & Argopecten purpuratus (Concha de abanico) & Ancón, Callao & 45 \\
Bivalvos & Gari solida (Almeja) & Huacho, Callao & 45 \\
TOTAL & Aulocomya ater (Choro) & Ancón, Huacho & 44 \\
& & & $\mathbf{2 5 4}$ \\
\hline
\end{tabular}

* Todas las especies se obtuvieron en el mercado pesquero de Ventanilla. 
Gráfico 1. Diagrama esquemático para el aislamiento, serotipificación y evaluación de patogenicidad de Vibrio parahaemolyticus

\section{COMPOSICIÓN \\ DE LA MUESTRA}

PREPARACIÓN

DE LA MUESTRA

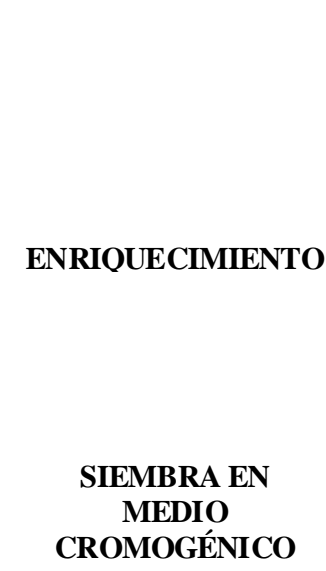

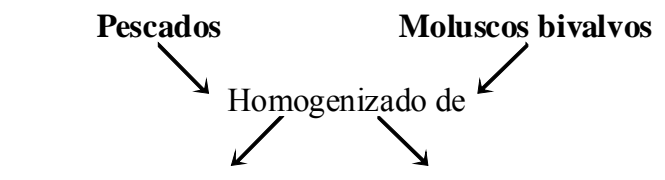

Piel y agallas

$25 \mathrm{~g}$ del homogenizado de pescado o marisco

$+$

$225 \mathrm{ml}$ de TSB 3\% NaCl

Incubar por 18 horas a $37^{\circ} \mathrm{C}$<smiles>C=[V]</smiles>

Extraer $1 \mathrm{ml}+9 \mathrm{ml}$ de caldo salado de Polimixina<smiles>[CH][CH]</smiles>

Incubar por 18 horas a $37^{\circ} \mathrm{C}$<smiles>[CH]1CC1</smiles>

Sembrar en CHROMagar Vibrio ${ }^{\mathrm{TM}}$ por dispersión y agotamiento.

Incubar por 18 horas a $37^{\circ} \mathrm{C}$<smiles>[CH]C</smiles>

Reaislar las colonias color malva presuntos V. parahaemolyticus en TSA $1.5 \% \mathrm{NaCl}$.

\section{VERIFICACIÓN \\ DEL \\ AISLAMIENTO}

puede inferir que la prevalencia de V.parahaemolyticus serotipo O3:K6 en la población de Odentesthes regia regia (Pejerrey) es de al menos $7 \%$.

Aunque no era el objetivo del trabajo, se realizó el seguimiento de otras colonias que desarrollaron en el medio cromogénico aislándose 71 cepas de Vibrio algynolyticus. No se aisló Vibrio cholerae ni $V$. vulnificus.

\section{DISCUSIÓN}

Vibrio parahaemolyticus se encuentra distribuido en ambientes marinos, costeros, estuarinos y en recursos 
Tabla 2. Distribución de Vibrio parahaemolyticus total, serotipo O3:K6 y fenómeno de Kanagawa positivo según grupo biológico.

\begin{tabular}{cccccc}
\hline $\begin{array}{c}\text { Grupo } \\
\text { biológico }\end{array}$ & Especie & $\begin{array}{c}\text { Aislamientos de Vibrio } \\
\text { parahaemolyticus } \\
\text { n (\%) }\end{array}$ & $\begin{array}{c}\text { Aislamientos } \\
\text { con Kanagawa } \\
\text { positivos } \\
\mathbf{n}(\%)\end{array}$ & $\begin{array}{c}\text { Serología + } \\
\text { O3 }\end{array}$ & K6 \\
\hline Pescados & $\begin{array}{c}\text { Jurel } \\
\text { Lisa }\end{array}$ & $1 / 40(2,5)$ & 0 & - & - \\
& Pejerrey & $2 / 40(5,0)$ & 0 & - & - \\
& $6 / 40(15,0)$ & $2 / 15(13,3)$ & $1 / 15$ & $1 / 15$ \\
Moluscos & $\begin{array}{c}\text { Conchas de } \\
\text { abanico }\end{array}$ & $2 / 45(4,4)$ & & & - \\
& Choro & $4 / 44(9,1)$ & $1 / 15(6,7 \%)$ & - & - \\
& Almeja & $0 / 45(0,0)$ & 0 & - & - \\
TOTAL & & $15 / 254$ & $3 / 15$ & $1 / 15$ \\
\hline
\end{tabular}

hidrobiológicos ya sea en forma avirulenta o virulenta. En este estudio se aisló en 5,9\% de las muestras analizadas. Estudios realizados en otras áreas geográficas reportan prevalencias desde $0,3 \%$ hasta $37,2 \%(15,16,21,22)$; resultados que evidencian su heterogénea distribución, siendo la cantidad que se puede aislar dependiente de la región, y probablemente relacionada con algún cambio en la naturaleza de la bacteria por factores abióticos y bióticos de repercusión global.

Asimismo, cabe resaltar que dicha especie no presenta distribución similar en los diferentes grupos biológicos. Nosotros encontramos una mayor frecuencia en pescados $(7,5 \%)$ que en moluscos bivalvos $(4,47 \%)$, resultados concordantes con los hallazgos obtenidos por Torres y Fernández (23). Otro estudio encontró que los moluscos bivalvos concentran en mayor frecuencia especies del género Vibrio aduciendo a que son filtradores de agua de mar (16); sin embargo, hay que tener en cuenta que en dicho estudio se analizó el contenido intestinal de los pescados donde la presencia de Vibrio parahaemolyticus depende de la última vez en la que el pez ingirió sus alimentos.

En nuestro estudio se evaluó el contenido de moluscos bivalvos, tejidos y agallas de pescados que también filtran agua de mar.

Llama la atención que el aislamiento de $V$. parahaemolyticus en pescados fue más frecuente en Odentesthes regia regia 15,0\% en comparación con las de otras especies estudiadas, este hallazgo discrepa con lo planteado por Liston (24) quien sugiere una distribución homogénea en las especies de peces de una misma región. Esto nos lleva a pensar que la distribución de dicha bacteria variaría de acuerdo a la profundidad en la que los recursos habitan (peces demersales, demersales costeros o pelágicos), depósitos, partículas en el agua, cantidad de material orgánico, temperatura, salinidad, época del año, entre otros.

El papel que ejerce la concentración de Vibrio parahaemolyticus en la generación de cuadros de gastroenteritis es cuestionable. En Estados Unidos y otros países de Europa a pesar que aseguran que los productos marinos para el consumo humano posean valores inferiores a $10^{4} \mathrm{UFC} / \mathrm{g}$, esta medida no ha evitado la aparición de brotes de diarrea aguda por esta especie de Vibrio (25). Por esta razón no se consideró en el objetivo de nuestro estudio.

Más importante que la concentración es la presencia de factores de virulencia como la hemolisina directa termoestable (TDH), factor que evaluamos en el agar de Wagatsuma buscando la expresión del gen tdh que se manifiesta con la presencia de â-hemólisis alrededor de las colonias productoras de TDH $(26,27)$. Tres $(20 \%)$ de los aislamientos presentaron fenómeno de Kanagawa positivo; este hallazgo es significativo dado que las cepas TDH + son las que se asocian a cuadros de gastroenteritis más severos (16).

Sólo una cepa con fenómeno de Kanagawa positivo presentó aglutinación con ambos antisueros, lo que representa el primer reporte en el Perú de la cepa pandémica de Vibrio parahaemolyticus O3:K6 a partir del análisis de una muestra de Odentesthes regia regia (Pejerrey), y confirma el consumo de alimentos marinos crudos o semicrudos como fuente de diarrea aguda, planteándose la necesidad de su inclusión en el sistema 
de vigilancia etiológica de enfermedad diarreica aguda (EDA).

Una característica de esta cepa pandémica es la gran morbilidad y producir desenlaces fatales en poblaciones especialmente propensas como inmunocomprometidos, gestantes, niños, ancianos, personas con gastritis, úlceras (4), infecciones por Helicobacter pylori que al alterar el $\mathrm{pH}$ gástrico favorece la acción de otros enteropatógenos como Vibrio parahaemolyticus (5).

Un brote de gastroenteritis por el serotipo O3: K6 en el Perú afectaría la salud de grandes grupos dado que el consumo de diversas formas de productos marinos por la población de la zona costera es muy común y en abundancia en la época de verano; aunado a cuantiosas perdidas económicas para el sector pesquero, pérdidas que afectarían por sobre todo a la pesca artesanal y atentaría contra el naciente prestigio del país de exportador de alimentos marinos de buena calidad.

Por esto es necesario establecer medidas preventivas para reducir la ocurrencia de enfermedad por este vibrio halofílico sobretodo en los meses estivales y durante el fenómeno del niño que se manifiesta cada 5 a 7 años (28).

Los planes de control que se aplican en otros países consisten en el conteo total indiferenciado de $V$. parahaemolyticus como el indicador para el control de la contaminación de alimentos dirigido a la prevención de infecciones; sin embargo, esta medida ha sido insuficiente para la prevención de brotes, sería entonces necesario no sólo determinar la concentración sino también evaluar periódicamente el potencial patógeno de las cepas colonizantes.

Otra manera efectiva de evitar estos brotes es por medio de campañas informativas a la población sobre el riesgo de la adquisición de enfermedades diarreicas por el consumo de productos marinos crudos o semicrudos e incluso marinados con limón en los que a pesar del bajo $\mathrm{pH}$ Vibrio parahaemolyticus ha podido ser aislado ya sea en pescados como en mariscos $(11,29)$.

Se plantea la necesidad de una evaluación de riesgo anual de $V$. parahaemolyticus en recursos hidrobiológicos de consumo humano por parte de los organismos de salud pública, así como continuar con la investigación en búsqueda de otros miembros del ahora llamado clon pandémico y el estudio molecular de los genes tdh y trh, para así tener un perfil genético de las cepas que colonizan nuestros recursos y conocer de antemano su potencial patógeno.

\section{Correspondencia:}

Jacqueline Vanessa Miranda Sanabria

Av. Las Magnolias 1023. Asoc. Viv. Res. Primavera. El Agustino

Lima, Perú

Correo electrónico: jacqueline.miranda@upch.pe

\section{REFERENCIAS BIBLIOGRÁFICAS}

1. Heitmann I, Jofré L, Hormázabal C, Olea A, Vallebuona $\mathrm{C}$, Valdés $\mathrm{C}$. Revisión y recomendaciones para el manejo de diarrea por Vibrio parahaemolyticus. Rev Chil Infect 2005; 22(2):131-140.

2. Lanata C. La enfermedad del cólera: a propósito de la primera pandemia en el Perú y América en este siglo. Salud Popular 1991; 3(1):7-25.

3. Gil A, Lanata C, Miranda H. Gravedad de la gastroenteritis causada por Vibrio parahaemolyticus del grupo pandémico en el Perú. Rev Peru Med Exp Salud Pública 2007; 24(4):350-355.

4. Hernandez C, Ulloa J, Vergara J, Espejo R, Cabello F. Infecciones por Vibrio parahemolyticus e intoxicaciones por algas: problemas emergentes de salud pública en Chile. Rev Med Chile 2005; 133(2):1081-1088.

5. Bag K, Nandi S, Bhadra R, Ramamurthy T, et al. Clonal diversity among recently emerged strains of Vibrio parahaemolyticus O3:K6 associated with pandemic spread. J Clin Microbiol 1999; 37(7):2354-2357.

6. Ibarra J, Delgado A, Alvarado D. Vibrios no Epidémicos y Vibrio cholerae O1 Asociados a Enfermedad Diarreica Aguda. Evento Climatológico "El Niño" - 1998. Hospital Nacional Dos de Mayo. An Fac Med (Perú) 1999;60(4): $251-256$.

7. Centers for Disease Control and Prevention. Outbreak of Vibrio parahaemolyticus infections association with eating raw oysters-Pacific Northwest 1997. Morb Mortal Wkly Rep 1998; 47(22):457-462.

8. Centers for Disease Control and Prevention. Outbreak eating raw oysters and clams harvested from Long Island Sound - Connecticut, New Jersey, and New York 1998. Morb Mortal Wkly Rep 1999; 48(03):48-51.

9. Infectious Diseases Surveillance Center, National Institute of Infectious Diseases. Vibrio parahaemolyticus, Japan, 1996-1998. Infect Agents Surv Rep 1999; 20:159160.

10. Colwell R. Global climate and infectious diseases: The cholera paradigm. Science 1996; 274(5295):2025-31.

11. Aliaga R. Aislamiento y caracterización de especies de Vibrio a partir de ceviches expendidos en la vía pública durante el fenómeno El Niño 1998. Tesis de Bachiller. 
Lima, Perú. Universidad Peruana Cayetano HerediaCiencias y Fisiología; 2005.

12. Gil A, Miranda H, Lanata C, et al. O3:K6 Serotype of Vibrio parahaemolyticus identical to the global pandemic clone associated with diarrhea in Peru. Int $\mathrm{J}$ Infect Dis 2007; 11: 324-328.

13. Instituto del mar del Perú .Boletín de la temperatura superficial del agua de mar en el litoral peruano. Disponible en URL: http://www.imarpe.gob.pe/imarpe/ archivos/boletines/imarpe_bltsm_tsm03_2009.pdf (Fecha de acceso: 01 marzo de 2009).

14. Ahlbom A, Norel S. Introduction to modern epidemiology. 2th ed. USA: Epidemiology Resources Inc; 1990. p. 24-29.

15. Vilcapoma A, Flores A, León J, Huaman L. Contaminación de Peces y mariscos por Vibriones Halofílicos en el Litoral Peruano. Santiago de Chile: Reunión de Emergencia sobre Cólera en el Pacifico Sudeste; 1991.p. 11.

16. Quintoil N, Porteen K, Pramanik A. Studies on occurrence of Vibrio parahaemolyticus in fin fishes and shellfishes from different ecosystem of West Bengal. Livest Res Rural Dev (serial online) 2007; 19(1). URL disponible en: http://www.lrrd.org/lrrd19/1/quin 19001.htm. (Fecha de acceso el 12 de diciembre del 2008).

17. Ministerio de la Producción - Oficina General de Tecnología de la Información y Estadística. Boletín Estadístico Mensual Noviembre 2008. URL disponible en:http://www.produce.gob.pe/portal/publicaciones.html. (Fecha de acceso el 12 de diciembre del 2008).

18. Elliot EL, Kaysner CA, Jackson L, Tamplin ML. Vibrio cholerae, V. parahaemolyticus, V. vulnificus and other Vibrio spp. En: AOAC International. Bacteriological Analytical Manual, Food and Drug Administration. 8th Edition. Gaithersburg, MD, USA: AOAC International; 1995. 2004. p.16-21.

19. Hara-Kudo Y, Sugiyama K, Nishibuchi M, et al. Prevalence of Pandemic Thermostable Direct
Hemolysin-Producing Vibrio parahaemolyticus O3:K6 in Seafood and the Coastal Environment in Japan. Appl Environ Microbiol 2003; 69 (7):3883-3891.

20. Nishibuchi M, Baper J. Thermostable Direct Hemolysin Gene of Vibrio parahaemolyticus: a Virulence Gene Acquired by a Marine Bacterium. American Society for Microbiology. 1995; 63: 2093 - 2099.

21. Kagiko M, Damiano A, Kayihura M. Characterisation of Vibrio parahaemolyticus isolated from fish in Kenya East Afr Med J 2001; 78 (3):124-7.

22. Ayres PA, Barrow GI. The distribution of Vibrio parahaemolyticus in British Coastal Waters: Report of a collaborative Study1975-6. J Hyg Camb. 1978; 80(2): 281-94.

23. Torres MR, Fernandez E. Incidence of Vibrio parahaemolyticus in Raw Fish, Oysters and Shrimp. Rev Latinoam Microbiol 1993; 35(3): 267-72.

24. Liston J, Milk J and Baross J. Distribution of Vibrio parahaemolyticus in the natural environment. Food Technol 1973; 36:113-117.

25. Daniels N, MacKinnon L, Bishop R, et al. Vibrio parahaemolyticus infections in the United States 1993-1998. J Infect Dis 2000; 181:1661-6.

26. Wagatsuma S. A medium for the test of the hemolytic activity of Vibrio parahaemolyticus. Media Circ 1968;13:159-162.

27. Shirai H, Ito H, Hirayama T, et al. Molecular epidemiologic evidence for association of thermostable direct hemolysin (TDH) and TDH-related hemolysin of Vibrio parahaemolyticus with gastroenteritis. Infect Immun 1990; 58:3568-73.

28. National Oceanic and Atmospheric Administration (NOAA). El Niño Page. URL disponible en: http:// www.elnino.noaa.gov/ (Fecha de acceso: 04 de julio del 2009).

29. Vilcapoma A, Flores A, León J, Alvarado D. Determinación de la frecuencia de Vibrio parahaemolyticus y otros vibriones halofílicos en alimentos preparados con productos marinos frescos y procesados. Rev Per Biol 1992; 4:17-20.

Recibido: 13/01/10

Aceptado para publicación: 19/08/10 